Conclusions: In those techniques with canal-wall techniques, canal-wall reconstruction techniques, or transcanal approaches, most surgical failures occur within the tympanic cavity and its hard-to-reach extensions, rather than within the mastoid. Using an endoscope enables us to see inside the facial recess, sinus tympani, and deep part of the round window niche, which are not fully visible under an operating microscope, thus leading to lower rates of cholesteatoma recurrence. These areas are minimally accessible even with extensive postauricular mastoidectomy. Microscopic surgery assisted by endoscope-guided dissection is therefore useful in such cases.

doi:10.1017/S0022215116006381

\section{ID: IP142}

Use of the apparent diffusion coefficient of conventional echo-planar imaging to differentiate between cholesteatomas and infectious lesions of the temporal bone

\section{Presenting Author: Hiroko Monobe}

Hiroko Monobe, Chikako Yamada, Kazunari Okada, Wakako Nakanishi, Miyako Ishii

Japanese Red Cross Medical Center

\section{Learning Objectives:}

Introduction: As therapeutic alternatives and technologies have advanced, the use of non-invasive modes of therapy to avoid surgery has increased. From this perspective the focus of this study was to evaluate the diagnostic benefit of the ADC in conventional echo-planar magnetic resonance imaging (MRI) as a means of differentiating between cholesteatomas and inflammatory lesions.

Methods: We evaluated three patients with suspected temporal-bone cholesteatomas, one infected cholesteatoma and three with inflammatory lesions by using MRI, including standard T2-weighted spin-echo and echo-planar DW/ ADC sequences, and computed tomography (CT) as aligned with regions of interest (ROIs) determined in DW imaging. The ADC values in the selected ROIs were calculated by using a 2-point linear regression method $(b=0$ and $b=1000 \mathrm{~s} / \mathrm{mm}^{2}$ ). To test the reliability, all measurements were performed twice; the coefficient of correlation was 0.94 .

Results: Three of the patients with suspected cholesteatoma and one patient with temporal-lobe abscessation due to temporal-bone inflammatory lesions subsequently underwent surgical confirmation and excision or drainage of their lesions. The ADC values were $0.759-0.915 \times 10^{-3} \mathrm{~mm}^{2} / \mathrm{s}$ (mean, $0.840 \times 10^{-3} \pm 0.0586 \mathrm{~mm}^{2} / \mathrm{s}$ ) for cases of uninfected cholesteatoma, $0.538-0.573 \times 10^{-3} \mathrm{~mm}^{2} / \mathrm{s}$ (mean, $0.555 \times 10^{-3} \pm 0.0141 \mathrm{~mm}^{2} / \mathrm{s}$ ) for infected cholesteatomas, and $0.905-1.272 \times 10^{-3} \mathrm{~mm}^{2} / \mathrm{s}$ (mean, $1.063 \times 10^{-3} \pm$ $0.123 \mathrm{~mm}^{2} / \mathrm{s}$ ) for inflammatory lesions. These ADC values differed significantly (one-way analysis of variance: $\mathrm{F}(2,11)=18.1, P<0.05)$.

Conclusions: The ADC value can be used preoperatively to differentiate between temporal-bone cbolesteatomas compared with infectious lesions. However, T2-weighted, FIESTA, or CISS images must be matched carefully to temporal-bone CT scans to accurately define ROIs.

doi:10.1017/S0022215116006393

\section{ID: IP143}

Surgical treatment for markedly advanced

petrous apex cholesteatoma via the

translabyrinthine and trans-sphenoidal

approach

\author{
Presenting Author: Tsunetaro Morino \\ Tsunetaro Morino $^{1}$, Kazuhisa Yamamoto ${ }^{1}$, \\ Yuichiro Yaguchi $^{2}$, Yutaka Yamamoto ${ }^{1}$, Hiromi Kojima ${ }^{1}$ \\ ${ }^{1}$ Jikei University School of Medicine, \\ ${ }^{2}$ St. Marianna University School of Medicine
}

Learning Objectives:

Introduction: Cholesteatoma is one of the most frequent lesions in the petrous portion of the temporal bone. It is difficult to completely remove the lesions of the petrous apex, specifically cholesteatoma. Selection of a surgical approach is determined by the location of the cholesteatoma in the petrous bone. We report a case of an intractable petrous apex cholesteatoma, which was managed with combined surgery, involving a translabyrinthine approach and transsphenoidal approach.

Case report: A 71-year-old Japanese man underwent right tympanoplasty 6 years previously. He visited our hospital because of recurrent cholesteatoma. He could not hear because of obstruction of the right external auditory meatus caused by the previous surgery. Computed tomography $(\mathrm{CT})$ revealed a soft tissue density lesion that extended widely from the petrous portion of the temporal bone to the sphenoid sinus. Cholesteatoma had extensively spread to the nearby carotid artery and posterior cranial fossa. It was considered difficult to completely remove this lesion. Surgery was performed via a translabyrinthine approach to remove most of the cholesteatoma. A trans-sphenoidal approach was used to create a drainage and observation hole. The postoperative course was uneventful, and the remainder of his clinical course was benign. Post-operative CT findings after 10 months revealed that the drainage route leads to the petrous portion of the temporal bone.

Conclusions: We encountered a markedly advanced, intractable petrous apex cholesteatoma. A surgical procedure, including trans-sphenoidal approach can maintain debris drainage and facilitate observation of the lesion from the 